\title{
Referees 2019
}

Published online: 13 February 2020

(C) Springer-Verlag GmbH Germany, part of Springer Nature 2020

We thank those who have given their time to referee for Diabetologia, all of whom are listed on our website (http://diabetologiajournal.org/about-the-journal/referees/). Special thanks are due to our key referees, listed below, who have each provided at least three timely and well-rated reports over the past year.

\author{
Alam U; Manchester \\ Amiel SA; London \\ Attie A; Madison \\ Bailey CJ; Birmingham \\ Bebu I; Rockville \\ Beck-Nielsen H; Odense \\ Biden TJ; Sydney \\ Biessels GJ; Utrecht \\ Bingley P; Bristol \\ Bjerre Knudsen L; Måløv \\ Blüher M; Leipzig \\ Bouzakri K; Strasbourg \\ Boyko E; Seattle \\ Buchanan TA; Los Angeles \\ Buscemi S; Palermo \\ Campbell-Thompson M; Gainesville \\ Carstensen B; Gentofte \\ Catalano P; Cleveland \\ Chaturvedi N; London \\ Chi L; Chapel Hill \\ Coles B; Leicester \\ Colhoun H; Edinburgh \\ Corpeleijn E; Groningen \\ Cox RD; Oxford \\ Dalgaard LT; Roskilde \\ Damm P; Copenhagen \\ Daniel C; Neuherberg \\ Devlin AM; Vancouver \\ DeVries JH; Amsterdam \\ Dhatariya K; Norwich \\ Donath MY; Zürich \\ Doucette C; Winnipeg \\ Dudding T; Bristol \\ Eckel J; Düsseldorf \\ Egan AM; Rochester
}

Ehses J; Fribourg

Eriksson O; Uppsala

Esguerra JLS; Malmö

Feig D; Toronto

Ferre P; Paris

Fujiyoshi A; Otsu

Gericke M; Halle

Gillespie K; Bristol

Glueck D; Aurora

Gorus FK; Brussels

Gottlieb PA; Denver

Green JB; Durham

Greenbaum CJ; Seattle

Gregg E; Atlanta

Gual P; Nice

Hamman RF; Denver

Hanson R; Phoenix

Harris RJ; London

Hastoy B; Headington

Hauguel-de Mouzon S; Cleveland

Haworth S; Bristol

Hernandez T; Aurora

Hill DJ; London

Hivert M-F; Boston

Hodson DJ; Birmingham

Holick M; Boston

Holst JJ; Copenhagen

Home P; Newcastle upon Tyne

Hughes RCE; Christchurch

Jeffcoate W; Nottingham

Johansen M; Baltimore

Johnson JD; Vancouver

Kadowaki T; Tokyo

Karpe F; Oxford

Kengne A; Cape Town
Kent S; Worcester

Koivula RW; Oxford

Kooijman S; Leiden

Kundu S; Nashville

Kushner JA; Houston

Lau B; Baltimore

Laybutt DR; Sydney

Lernmark Å; Malmö

Leslie RDG; London

Long A; Seattle

Lystig T; Ridgefield

Ma RCW; Hong Kong

Maechler P; Geneva

Malik RA; Doha

Mallone R; Paris

Mannering SI; Melbourne

Margolis D; Philadelphia

Marques AC; Lausanne

McIntyre D; Queensland

McNeilly A; Dundee

Merino J; Boston

Mingrone G; Rome

Mitchell BD; Baltimore

Morris K; Cardiff

Mulder H; Malmö

Murphy HR; Norwich

Narayan KMV; Atlanta

Nascimento E; Maastricht

Nichols GA; Portland

Nijpels G; Amsterdam

Nirantharakumar K; Birmingham

Noordam R; Leiden

Orešič M; Turku

O’Tierney-Ginn P; Boston

Packer M; Dallas 
Pan Q; Washington

Patel C; Boston

Pavkov ME; Atlanta

Pearson E; Dundee

Perng W; Aurora

Peters S; Oxford

Petrie JR; Glasgow

Piemonti L; Milano

Piro S; Catania

Pociot F; Herlev

Poston L; London

Prieur X; Nantes

Retnakaran R; Toronto

Rickels MR; Philadelphia

Rodriguez Calvo T; Munich

Romeo S; Gothenburg

Rooney M; Baltimore

Rossing P; Gentofte

Ruiz-Canela M; Pamplona
Rutter MK; Manchester

Sattar N; Glasgow

Sauder K; Aurora

Saydah SH; Silver Spring

Scott EM; Leeds

Selvarajah D; Sheffield

Shafi T; Jackson

Shapiro A; Aurora

Sharp S; Cambridge

Shaw J; Melbourne

Simmons D; Campbelltown

Simmons RA; Philadelphia

Sims EK; Indianapolis

Skog O; Uppsala

Solimena M; Dresden

Stefan N; Tübingen

Stene LC; Oslo

Stevens RJ; Oxford

Stewart AF; New York
Stienstra R; Nijmegen

Tahrani A; Birmingham

Tse H; Birmingham

Twigg SM; Sydney

Umpierrez G; Atlanta

van de Bunt $\mathrm{M}$; Oxford

van Zuydam N; Oxford

Verhagen J; London

Virtue S; Cambridge

Vos R; The Hague

Wander PL; Seattle

Watada H; Tokyo

Wen L; New Haven

Wheeler E; Cambridge

Williams AJK; Bristol

Woodward M; Sydney

Young R; Glasgow

Zhang C; Rockville

Ziegler D; Düsseldorf

Publisher's note Springer Nature remains neutral with regard to jurisdictional claims in published maps and institutional affiliations. 